\title{
Programa Bolsa Família e saneamento: uma revisão sistemática dos efeitos na diarreia e na desnutrição
}

\author{
Bolsa Família Program and environmental health: a systematic \\ review of the effects on diarrhea and malnutrition
}

Anelise Andrade de Souza (https://orcid.org/0000-0002-2408-7054) ${ }^{1}$

Léo Heller (https://orcid.org/0000-0003-0175-0180) ${ }^{1}$

${ }^{1}$ Instituto René Rachou, Fundação Oswaldo Cruz. Av. Augusto de Lima 1715, Barro Preto. 30190-002 Belo Horizonte MG Brasil. asouzandrade@

yahoo.com.br

\begin{abstract}
The Bolsa Familia Programme and environmental health interventions are public policies that can have a combined positive effect on health inequities. The Bolsa Familia Programme is designed to improve health conditions, reduce food insecurity and increase family incomes. Environmental health interventions aim to ensure public health and environmental protection. This study reviewed the literature for possible interactions between these two types of intervention that influence morbidity and mortality outcomes due to diarrhoea and malnutrition in the under-fives. A total of 1,658 articles were identified in the LILACS, SciELO and PubMed databases. The studies' methodologies were evaluated by scores on an adapted Downs \& Black scale and four met all the study inclusion criteria. The findings showed evidence of the positive independent effects of the Bolsa Familia Programme and of environmental health interventions in reducing illness and death from diarrhoea and malnutrition in the study age group. However, none of the articles offered results that might elucidate a joint effect of these public policies on an interaction model, revealing a gap in the literature on these diseases attributable particularly to poverty.

Key words Systematic review, Environmental health interventions, Social programmes. Public policy
\end{abstract}

Resumo O Programa Bolsa Família e intervenções em saneamento são políticas públicas que podem ter efeito combinado positivo no enfrentamento das iniquidades em saúde. O Programa Bolsa Família busca propiciar melhoria das condições de saúde, diminuição da insegurança alimentar e aumento da renda familiar. Intervenções em saneamento objetivam garantir a saúde pública e a proteção do meio ambiente. O estudo objetivou revisar a literatura, procurando indícios de interações entre estas duas intervenções, influenciando nos desfechos morbidade e mortalidade por diarreia e desnutrição em menores de cinco anos. Foi realizada busca nas bases LILACS, SciELO e PubMed e a metodologia dos artigos foi avaliada por meio de escores da escala de Downs \& Black adaptada. Foram identificados 1.658 artigos, dos quais quatro apresentaram todos os critérios para inclusão no estudo. Os estudos selecionados mostraram evidências do efeito positivo do Programa Bolsa Família e de intervenções em saneamento relacionados aos desfechos de interesse, quando analisados de forma independente um do outro. No entanto, nenhum artigo apresentou resultados que pudessem esclarecer o efeito conjunto destas políticas públicas em um modelo de interação, revelando lacuna na literatura nestas doenças particularmente atribuíveis à pobreza.

Palavras-chave Revisão sistemática, Saneamento, Programas sociais, Políticas públicas 


\section{Introdução}

Os Programas de Transferência Condicionada de Renda (PTCR) surgiram com o intuito de romper o ciclo intergeracional da pobreza, fornecendo para as famílias pobres uma fonte de renda regular mínima, condicionada à sua participação em serviços ofertados pelo estado, em geral nas áreas da educação e saúde ${ }^{1}$ O Programa Bolsa Família (PBF), um PTCR brasileiro, foi criado no ano de 2003 e regulamentado em 2004, por meio da Lei no ${ }^{\circ} 10.836 / 2004^{2}$ e do Decreto n. ${ }^{\circ} 5.209 / 2004^{3}$, com base na fusão progressiva de quatro diferentes programas de transferência de renda (Bolsa Escola, Bolsa Alimentação, Cartão Alimentação e Auxílio Gás) ${ }^{2}$. Atende famílias em situação de pobreza e extrema pobreza, e se baseia em três distintas dimensões: transferência de renda, condicionalidades nas áreas de saúde e educação e ações complementares, que se referem a outros programas sociais que somente famílias cadastradas no Cadastro Único para Programas Sociais do Governo Federal (CadÚnico) podem ter aces$\mathrm{so}^{2}$. Dessa forma, o PBF busca propiciar a inclusão social das famílias beneficiárias tornando-se ferramenta de oportunidades e emancipação das condições de pobreza, melhoria das condições de saúde, diminuição da insegurança alimentar e aumento da renda familiar ${ }^{4}$.No ano de sua criação, o PBF atendeu 3,6 milhões de famílias, atingindo nos anos de 2013 e 2018, 14,1 e 14,2 milhões de famílias beneficiárias, respectivamente. Em 2019, o Programa beneficiou 13,8 milhões de famílias que receberam por mês, em média, 186,78 reais $^{5}$.

Em relação ao saneamento, nos últimos anos, as principais orientações que vêm regulando o setor estão representadas pela Lei $\mathrm{n}^{\circ} 11.445 / 2007^{6}$. Essa legislação estabelece as diretrizes nacionais para o saneamento básico no Brasil e tem como objetivo a cobertura universal a serviços adequados de água, esgotamento sanitário, limpeza urbana e coleta de resíduos sólidos. Segundo dados do Plano Nacional de Saneamento Básico $(\text { PLANSAB })^{7}$, em 2017, 85, 79\% dos domicílios brasileiros estavam ligados à rede de abastecimento de água, chegando a 95,6\% se incluído o abastecimento via água de poço.No entanto, $40 \%$ da população não atendida, no período de 2007 a 2015, correspondeu à faixa de renda de até um salário mínimo e do universo da população com acesso à rede pública, $86,7 \%$ não tinham água diariamente ${ }^{8}$. Em relação ao esgotamento sanitário, os números mostram que o Brasil está longe da universalização, com apenas $66,5 \%$ dos domicílios ligados à rede geral de esgoto ou plu- vial e $15,6 \%$ utilizando fossa séptica no ano de $2017^{7}$. Além disso, embora tenha ocorrido uma evolução positiva de $6,8 \%$ no tratamento de esgoto desde o ano de 2013, apenas 50\% do total de volume de esgotos gerados no ano de 2016 pela população foram tratados no Brasil ${ }^{8}$.

$\mathrm{O}$ PBF e as intervenções em saneamento são políticas públicas que podem ter efeito muito positivo no enfrentamento das iniquidades em saúde, sobretudo quando as duas intervenções são associadas. Ao longo dos anos de vigência do PBF no Brasil, estudos apontam redução da mortalidade em menores de cinco anos de idade e mortalidade infantil relacionada ao aumento da cobertura da população pelo Programa ${ }^{9-11}$, assim como melhoria do estado nutricional dos beneficiários e diminuição de morbidade por desnutrição e diarreia ${ }^{11-19}$. Outros estudos apontam associação entre melhores condições de saneamento da população, como disponibilidade de acesso à água e esgotamento sanitário, com melhores resultados em saúde end,20-24. $^{13}$

Dado o contexto apresentado, surge a indagação de se a presença simultânea de implementação de bolsa família e de melhores condições de saneamento potencializariam melhores resultados em saúde. Assim, teve-se como objetivo neste estudo, revisar a literatura, buscando indícios de possíveis interações entre as duas intervenções, influenciando os desfechos morbidade e mortalidade por diarreia e desnutrição em crianças menores de cinco anos de idade. E dessa forma, subsidiar investigações futuras, descrevendo o atual estado da arte referente a esta temática.

\section{Métodos}

Trata-se de uma revisão sistemática da literatura científica de trabalhos publicados, que utilizou para sua concepção as recomendações propostas pela Cochrane Collaboration ${ }^{25}$ e pelo Preferred Reporting Items for Systematic Reviews and Meta-Analysis (PRISMA) ${ }^{26}$, apresentando como questão norteadora da pesquisa: $O$ acesso conjunto ao $P B F$ e a condições adequadas de saneamento resultam em melhores resultados em saúde, relacionados à morbidade e mortalidade por diarreia e desnutrição em crianças na faixa etária abaixo de cinco anos? Para responder a esse questionamento, as seguintes etapas foram realizadas $\left.{ }^{27}: 1\right)$ Identificação do problema de revisão; 2) Formulação da questão norteadora; 3) Seleção da amostra; 4) Categorização e análise dos dados; 5) Discussão dos resultados e síntese do conhecimento. 
A coleta de dados foi realizada no período de outubro a novembro de 2019, por meio de pesquisas nas bases de dados eletrônicas da Literatura Latino Americana e do Caribe (LILACS), National Library of Medicine - National Institutes of Health (PubMed), ScientificElectronic Library Online (SciELO), além de buscas manuais em periódicos citados nas referências dos artigos selecionados. Foram adotados descritores nos idiomas inglês, espanhol e português, combinados entre si com auxílio de operador booleano (AND), sendo eles: "bolsa família", "programa de transferência condicionada de renda", "saneamento", "acesso à água", "esgotamento sanitário", "diarreia", "desnutrição". A seleção dos estudos restringiu-se a trabalhos publicados entre outubro de 2003 (criação do PBF) e novembro de 2019.

Os artigos localizados foram submetidos ao seguinte critério de inclusão: artigos completos e com acesso livre nos idiomas inglês, espanhol e português que avaliaram o PBF e acesso ao saneamento com resultados em saúde relacionados à mortalidade e/ou morbidade por diarreia e desnutrição. Os artigos localizados foram submetidos posteriormente aos seguintes critérios de exclusão: 1) artigos repetidos nas bases de dados e/ou duplicados dentro da mesma base de dados; 2) artigos que avaliaram indivíduos fora da faixa de interesse do estudo, sendo denominado exclusão de acordo com o tipo de população (TP); 3) artigos de revisões sistemáticas e integrativas, além de estudos de caso, sendo denominado exclusão de acordo com tipo de estudo(TE); (4) artigos que abordaram outros PTCR, que não fosse o PBF, além de desfechos diferentes de diarreia e desnutrição, por sua vez, denominado exclusão de acordo com o tipo de intervenção (TI).Os artigos foram selecionados primeiramente por meio da leitura do título e do resumo do artigo, de forma a visualizar se possuíam características referentes à pergunta norteadora da pesquisa. Aqueles que possuíam informações que respondiam ao questionamento da atual pesquisa foram lidos na íntegra.

Os dados dos artigos selecionados foram coletados de forma independente utilizando uma planilha do programa Microsoft Office Excel 2010, registradas as seguintes informaçoes: autoria, ano e periódico de publicação, título, unidade de análise (nacional, regional ou local), tipo de estudo, grupo de comparação, variáveis dependentes e independentes de interesse do atual estudo,medidasestatísticas, medidas de associação e Intervalos de Confiança (IC) quando disponí- veis e presença ou não de impactos do PBF e do saneamento nos desfechos de interesse.

Além disso, os artigos finais selecionados foram avaliados segundo a sua qualidade metodológica, utilizando o método validado de Downs\& Black $(1998)^{28}$, adaptado para estudos não experimentais, com utilização de 20 itens de avaliação: objetivos/hipótese descritos; clareza na apresentação dos resultadosna seção de resumo; descrição na seção de metodologia dos principais desfechos; definição dos critérios de inclusão e exclusão;descrição das intervenções de interesse, quando havia; lista dos principais fatores de confusão; descrição dosachados do estudo edas estimativas da variabilidade destes resultados; descrição, quando havia, da perda de acompanhamento; valores reais de probabilidade reportados integralmente; amostra com representatividade; mesmo tempo de seguimento para todos os participantes do estudo, quando couber; adequabilidade dos testes estatísticos; medidas para avaliar o desfecho adequadas e confiáveis; indivíduos recrutados na mesma população e mesmo período, quando couber; ajuste adequado para fatores de confusão; limitações do estudo definidas; perdas de indivíduos, municípios ou unidades de análise reportadas e, apresentação do poder de efeito do estudo. Para cada uma dessas categorias foi computado o valor "1" quando presente o critério avaliado e " 0 " quando ausente. Nos casos de não aplicação do critério, o valor computado do escore foi de " 1 ". Quanto mais próximo do valor total (20/100\%) melhor a qualidade do estudo.

\section{Resultados}

\section{Caracterização dos estudos}

A Figura 1 ilustra as etapas de seleção para a estruturação da revisão, em que foram identificados 1.658 artigos, sendo 1.613 com disponibilidade de texto completo. Desses 1.613 artigos, 1.611 foram selecionados nas três diferentes bases de dados utilizadas no estudo e dois resultantes de uma busca manual. Ao aplicar os critérios de exclusão dos artigos selecionados, foram excluídos: 785 (48,66\%) artigos duplicados; 468 (29,02\%) após avaliação do título; $286(17,73 \%)$ após avaliação do resumo e 70 (4,33\%) após leitura do artigo integralmente. Dessa forma, quatro $(0,26 \%)$ artigos atenderam aos critérios de inclusão e exclusão e foram utilizados como resultado desta revisão. Destes estudos, dois foram publicados na língua inglesa e dois na língua portuguesa. 


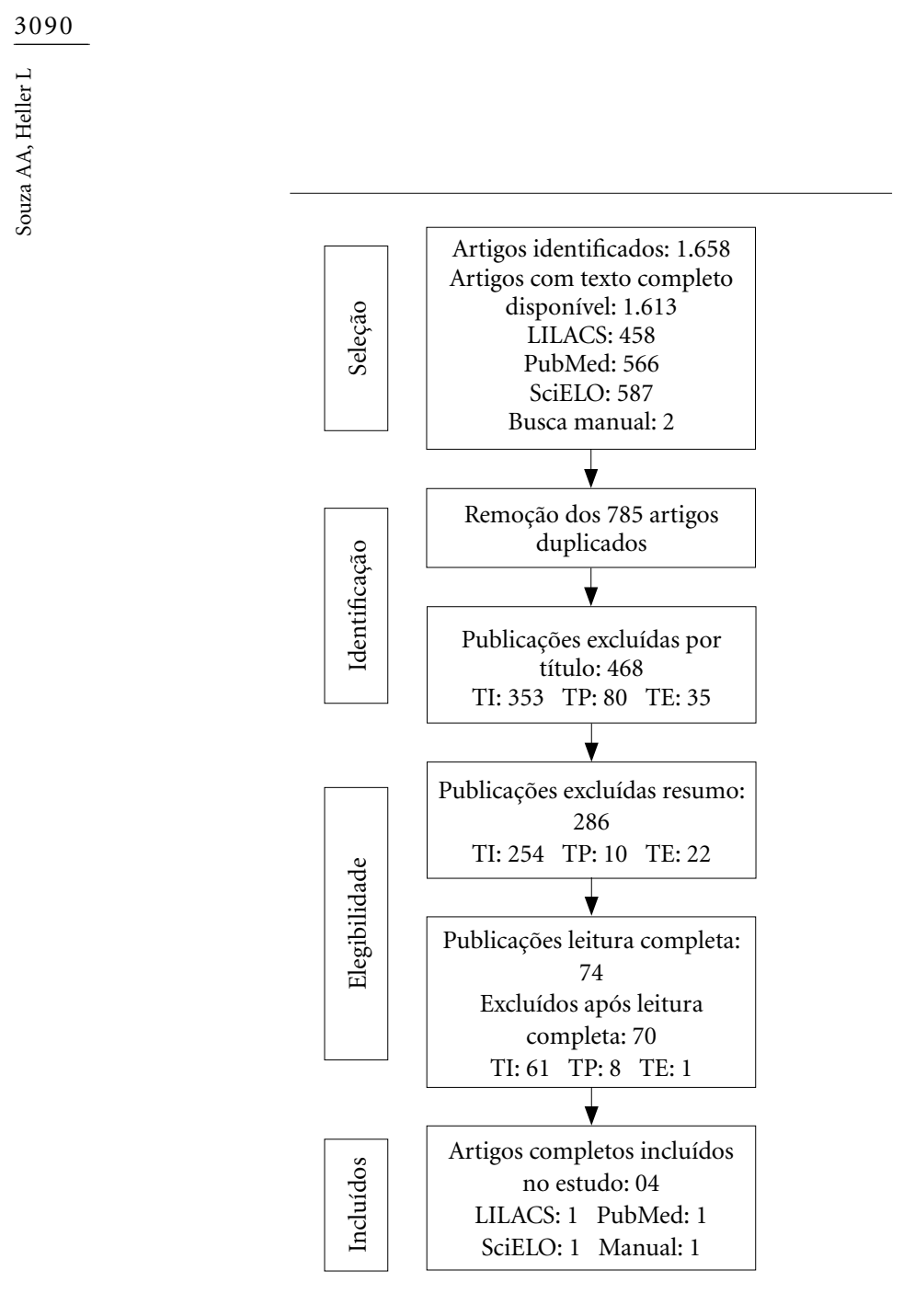

Figura 1. Fluxograma das etapas de seleção para estruturação de estudos da revisão sistemática.

TI: Tipo de Intervenção. TP: Tipo de População. TE: Tipo de Estudo.

Fonte: Elaborado pelos autores.

O Quadro 1 traz as características de interesse dos estudos incluídos nesta revisão. Após a seleção destes artigos foram extraídas informações de acordo com as variáveis mencionadas na seção de metodologia. Todos os artigos utilizaram a abordagem metodológica quantitativa e o período de referência variou de 2004 a 2012. Os artigos foram publicados em periódicos nacionais (2) e internacionais (2) e, quanto a abrangência geográfica, um deles utilizou uma amostra dos municípios brasileiros ${ }^{11}$, dois deles relacionaram-se a estudos locais ${ }^{13,15}$, e um, avaliou macrorregiões brasileiras ${ }^{17}$. Em relação às variáveis de interesse, todos os estudos consideraram o saneamento e o PBF como variáveisindependentes. Para o sa- neamento, a forma como foi tratado nos estudos mostrou-sevariável. Rasella et al. ${ }^{11}$ e Chagas et al. ${ }^{15}$ utilizaram a variável de forma agregada (acesso à água, esgotamento sanitário e coleta de resíduos sólidos), classificando os municípios em acesso adequado ou inadequado. Paes-Sousa, Santos e Miazaki ${ }^{17}$ avaliaram somente o acesso à água e Imada et al..$^{13}$ avaliaram separadamente a presença de vaso sanitário domiciliar, procedência da água, frequência de falta de água no domicílio, existência de tratamento da água de beber e destino dos resíduos sólidos e do esgoto. Em relação ao PBF, três estudos utilizaram como análise, grupos de comparação entre exposição ou não ao Programa ${ }^{13,15,17}$, e um, avaliou a proporção de cobertura da população alvo e proporção de cobertura da população total municipal ${ }^{11}$.

Em relação às variáveis de desfecho de interesse do presente estudo, um estudo avaliou morbidade e mortalidade por diarreia e desnutrição ${ }^{11}$, dois avaliaram morbidade por desnutrição ${ }^{15,17}$, e outro, morbidade por diarreia ${ }^{13}$.

Por fim, a Tabela 1 apresenta os dados referentes à avaliação da qualidade metodológica dos artigos incluídos nesta revisão, segundo metodologia de Downs \& Black ${ }^{28}$ adaptada. Foi considerado um total de 20 critérios, e dessa forma, valor máximo de 20 pontos, relacionados a quatro grupos: características dos estudos, validade interna, validade externa e poder do efeito do estudo. A mediana do escore encontrada foi de 16,5 pontos, com o valor mínimo de 13 e máximo de 17 pontos. A maioria dos estudos apresentou pontuação máxima entre 16 e 17 pontos (75\%). As principais limitações relacionadas aos estudos dizem respeito a descrição dos fatores de confusão e seu ajuste adequado, definição das limitações e relato claro das perdas de indivíduos/municípios ou suas unidades de análise, seguidos pela descrição dos valores reais das probabilidades e representatividade da amostra. Todos os demais critérios foram avaliados com valores de escores entre $75 \%$ e $100 \%$.

\section{Associações com morbidade e mortalidade por diarreia e desnutrição}

Rasella et al. ${ }^{11}$ encontraram efeito protetivo do PBF em relação a internações e mortes por diarreia e desnutrição. A variável PBF foi tratada de acordo com dois parâmetros: cobertura da população total do município pelo Programa (CPT) e cobertura da população alvo peloPrograma (CPA). A partir dessa categorização, os municípios foram classificados em quatro 


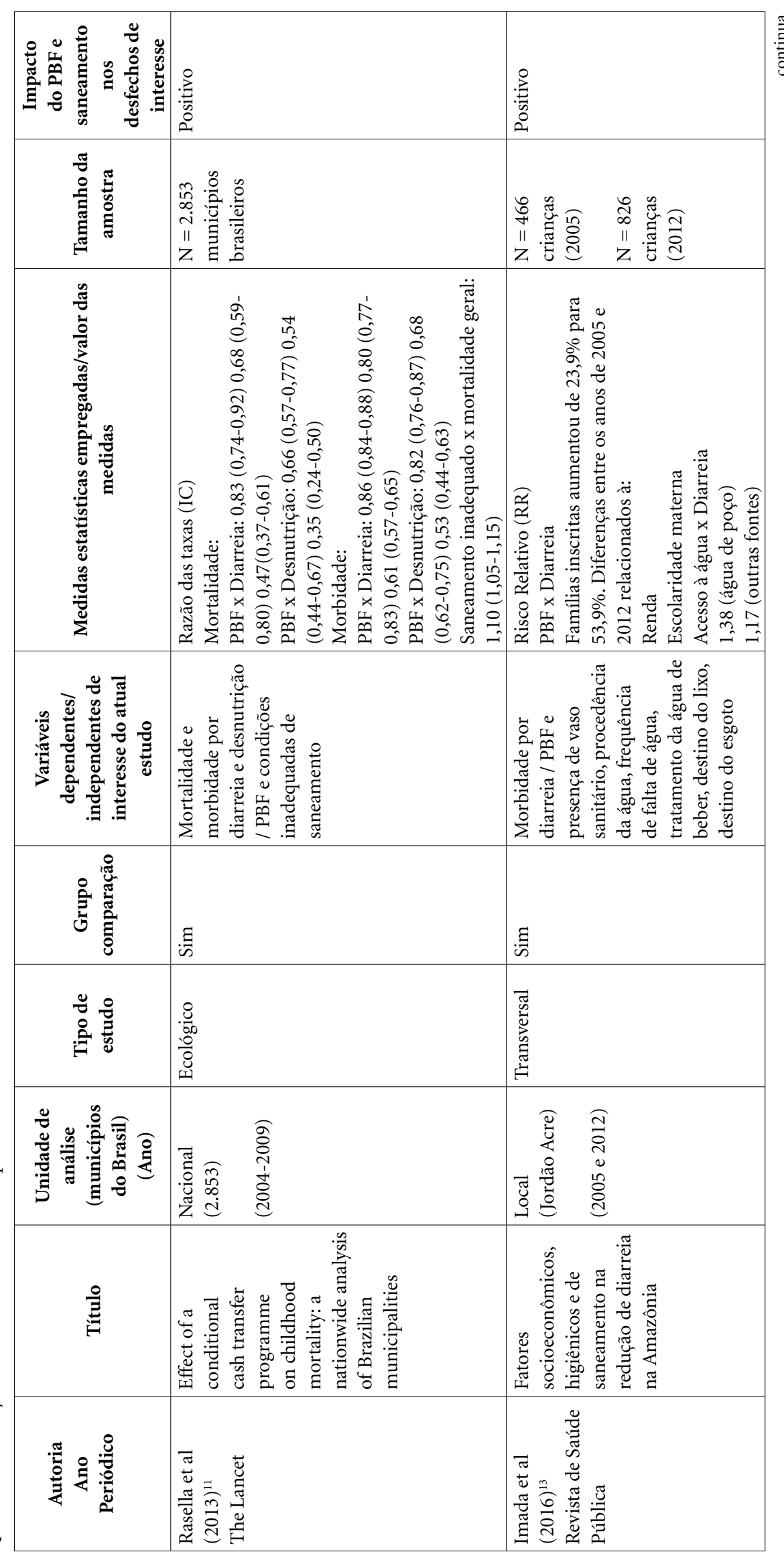




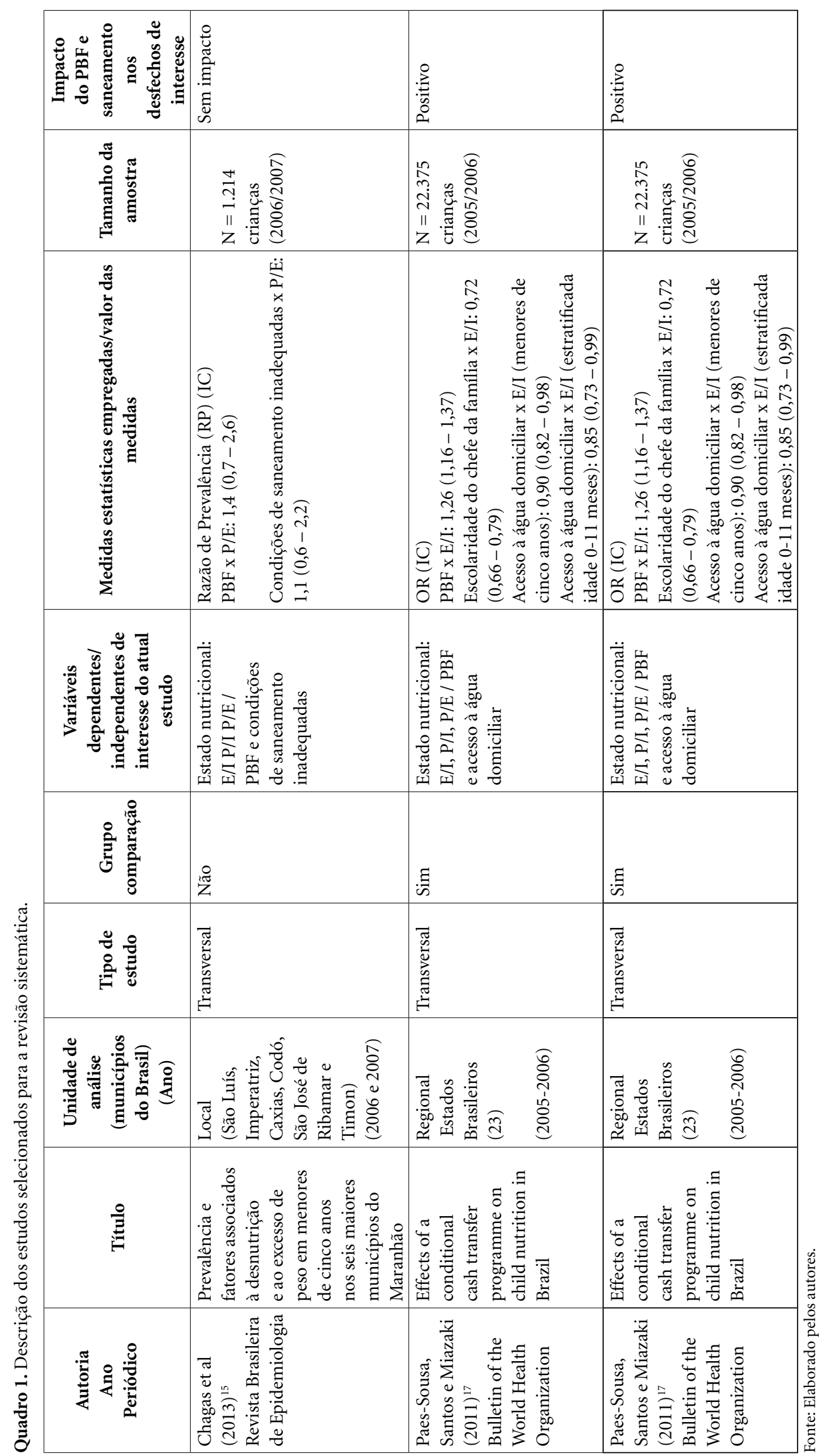


Tabela 1. Percentual de acertos segundo os critérios da escala adaptada de Downs\& Black $(1998)^{28}, \mathrm{n}=4$.

\begin{tabular}{|c|c|}
\hline Critérios & $\begin{array}{c}\text { Acertos } \\
(\%)\end{array}$ \\
\hline \multicolumn{2}{|l|}{ Características do estudo } \\
\hline Objetivos/hipótese claramente descritos & 100,0 \\
\hline $\begin{array}{l}\text { Os principais resultados do estudo são } \\
\text { apresentados claramente na seção do } \\
\text { resumo? }\end{array}$ & 75,0 \\
\hline $\begin{array}{l}\text { Desfechos principais medidos descritos na } \\
\text { metodologia }\end{array}$ & 100,0 \\
\hline $\begin{array}{l}\text { Definição dos critérios de inclusão e } \\
\text { exclusão no estudo }\end{array}$ & 75,0 \\
\hline $\begin{array}{l}\text { As intervenções de interesse, quando } \\
\text { aplicadas no estudo, estão claramente } \\
\text { descritas? }\end{array}$ & 100,0 \\
\hline $\begin{array}{l}\text { É fornecida uma lista dos principais } \\
\text { fatores de confusão? }\end{array}$ & 25,0 \\
\hline $\begin{array}{l}\text { Principais achados/desfechos do estudo } \\
\text { claramente descritos }\end{array}$ & 100,0 \\
\hline $\begin{array}{l}\text { O estudo fornece estimativas da } \\
\text { variabilidade dos dados, para os principais } \\
\text { resultados? }\end{array}$ & 100,0 \\
\hline Perdas de acompanhamento descritas? & 100,0 \\
\hline $\begin{array}{l}\text { Valores reais das probabilidades } \\
\text { reportadas integralmente? }\end{array}$ & 75,0 \\
\hline \multicolumn{2}{|l|}{ Validade externa } \\
\hline Representatividade da amostra & 75,0 \\
\hline $\begin{array}{l}\text { Tempo de seguimento igual para toda a } \\
\text { amostra, quando couber }\end{array}$ & 100,0 \\
\hline Testes estatísticos apropriados & 100,0 \\
\hline Medidas de desfecho válidas e confiáveis & 100,0 \\
\hline \multicolumn{2}{|l|}{ Validade interna } \\
\hline $\begin{array}{l}\text { Indivíduos recrutados na mesma } \\
\text { população, quando couber }\end{array}$ & 100,0 \\
\hline $\begin{array}{l}\text { Indivíduos recrutados no mesmo período, } \\
\text { quando couber }\end{array}$ & 100,0 \\
\hline $\begin{array}{l}\text { Ajuste adequado para fatores de confusão } \\
\text { na análise }\end{array}$ & 25,0 \\
\hline $\begin{array}{l}\text { Limitações do estudo claramente } \\
\text { definidas }\end{array}$ & 25,0 \\
\hline $\begin{array}{l}\text { Perdas de indivíduos/municípios/ } \\
\text { unidades de análise reportados }\end{array}$ & 25,0 \\
\hline \multicolumn{2}{|l|}{ Poder do estudo } \\
\hline Poder de efeito do estudo $(\mathrm{p}<0,05)$ & 100,0 \\
\hline
\end{tabular}

categorias: cobertura baixa (0\%-17,1\% CPT), intermediária $(17,2-32,0 \% \mathrm{CPT})$, alta $(>32,0 \%$ CPT) e consolidada ( $>32,0 \%$ CPT e $>100,0 \%$ CPA nos últimos 4 anos). O efeito protetivo em relação à morbidade por diarreia e desnutrição aumentou na medida em que a cobertura evoluiu de baixa a consolidada, sendo a razão das taxase seus respectivosIC para diarreia nas coberturas intermediária, alta e consolidada, 0,86 (IC 0,840,78 ); 0,80 (IC $0,77-0,83$ ) e 0,61 (IC $0,57-0,65$ ) e para desnutrição, 0,82 (IC 0,76-0,87); 0,68 (IC 0,62-0,75) e 0,53 (IC 0,44-0,63). Resultado semelhante foi observado quando avaliada também, a mortalidade por estas causas, sendo a razão das taxas para diarreia, 0,83 (IC 0,74-0,92); 0,68 (IC $0,59-0,80)$ e 0,47 (IC $0,37-0,61)$ e para desnutrição, 0,66 (IC 0,57-0,77); 0,54 (IC 0,44-0,67) e 0,35 (IC 0,24-0,50). Também houve associação quando presentes condições inadequadas de saneamento básico, com razão das taxas de 1,10 (IC 1,05-1,15), ou seja, municípios que apresentaram cobertura de serviços de saneamento abaixo da mediana apresentaram maiores riscos relacionados à mortalidade por todas as causas, em crianças na faixa etária de interesse do estudo. Os autores concluíram que um PTCR, como o PBF, pode contribuir significativamente para a diminuição de morbidade e mortalidade em crianças menores de cinco anos por todas as causas e, em particular, por mortes atribuíveis a causas relacionadas à pobreza, como desnutrição e diarreia.

\section{Associação com morbidade por diarreia}

Imada et al. ${ }^{13}$ avaliaram morbidade por diarreia a partir da comparação de resultados de dois estudos transversais realizados nos anos de 2005 e 2012, em um município do Acre, considerado um dos municípios mais pobres do país. A comparação dos resultados dos dois inquéritos mostrou diminuição da prevalência da doença $(45,1 \%$ para 35,4\%), mas aumento da internação por esta causa $(4,5 \%$ para $10,7 \%)$, principalmente na zona urbana do município. Os autores encontraram associação significativa para o desfecho diarreia quando avaliado o acesso à água, sendo protetiva a água proveniente da rede pública, com Risco Relativo (RR) de 1,38 se proveniente de poço ou rio e $R R$ de 1,17 se proveniente de outras fontes. Entre os anos que transcorreram entre os dois estudos, ocorreram melhorias nas condições de moradia da população, aumento do número de vasos sanitários domiciliares de cerâmica, melhoria no acesso a água e destino do esgoto. Os autores concluíram que as condições de saneamento no município apresentaram melhorias expressivas, mas o abastecimento de água e o esgotamento sanitário ainda não são universais, com ônus principalmente para a população rural.

Os resultados do estudo também mostram o efeito do PBF, que teve aumento da proporção de famílias inscritas entre os dois anos de análise $(23,9 \%$ para $53,9 \%)$, observado por meio de 
suas dimensões e condicionalidades: aumento da renda familiar, considerada fator de incremento para aquisição de bens de consumo e alimentos de melhor qualidade e, melhoria do acesso à educação, resultando em diminuição do analfabetismo, de $17,4 \%$ para $12,0 \%$, com aumento da escolaridade materna.

\section{Associação com morbidade por desnutrição}

Estudo realizado por Chagas et al. ${ }^{15} \mathrm{em}$ seis municípios do Maranhão, Brasil, objetivou avaliar as prevalências e os fatores associados à desnutrição e ao excesso de peso em crianças menores de cinco anos de idade. A prevalência de desnutrição foi de 8,5\%, segundo Estatura por Idade (E/I); 3,9\%, segundo Peso/Estatura (P/E) e $4,5 \%$ segundo Peso por Idade $(\mathrm{P} / \mathrm{I})$. No modelo de regressão logística para $\mathrm{P} / \mathrm{E}$ (único indicador com resultados apresentados), não houve associação entre esse indicador e o PBF (Razão de Prevalência/RP 1,4, IC 0,7-2,6), assim como não houve associação com exposição a condições inadequadas de saneamento (RP 1,1, IC 0,6-2,2). Os autores concluem igualdade social em relação à desnutrição, sugerindo evolução favorável dos municípios no sentido de maior equidade.

Paes-Sousa, Santos e Miazaki ${ }^{17}$ avaliaram dados de 22.375 crianças menores de cinco anos, de 479 municípios brasileiros, referentes a 23 estados. As crianças foram divididas em grupos expostos e não expostos ao PBF e avaliadas segundo seu estado nutricional (EN). Os resultados do estudo mostraram diferença significativa entre os grupos expostos e não expostos, quando avaliado déficit nutricional em relação à estatura/idade (E/I) e peso/idade (P/I), sendo que as crianças inscritas no PBF tiveram uma chance $26 \%$ maior (OddsRatio/OR 1,26 IC 1,16-1,37) de terem E/I adequada. Escolaridade do chefe de família, um reflexo das condicionalidades do $\mathrm{PBF}$, também se mostrou protetiva em relação a E/I (OR 0,72, IC 0,66-0,79), assim como o acesso da população do estudo à água domiciliar (OR 0,90, IC 0,82-0,98). Quando estratificado por idade, acesso à água domiciliar e E/I apresentaram associação na faixa etária de zero a onze meses (OR 0,85, IC 0,730,99). Os autores discutem a importância do PBF relacionado ao aumento da renda dos beneficiários, que proporciona melhor acesso a alimentos, bens e serviços. Além disso, discutem a importância das condicionalidades em educação que permitiram aumento da escolaridade da população brasileira ao longo da existência do Programa, e consequentemente cuidados mais adequados às crianças. Melhores estruturas de serviços sociais, incluindo acesso à água, esgotamento sanitário e coleta de resíduos sólidos, por meio de políticas de saneamento também são importantes para resultados melhores em saúde.

\section{Discussão}

Os PTCR, como o PBF, assim como políticas públicas que objetivam melhorias do ambiente físico, como intervenções em saneamento, são ações que necessitam ter sua efetividade avaliada, por serem estratégias relevantes para confrontar os riscos de deterioração das condições sociais no atual cenário nacional e para receberem os aprimoramentos necessários. O Brasil apresenta famílias em situação de vulnerabilidade econômica e social, associadas à extrema pobreza estrutural, caracterizada pelo subemprego, baixos salários, falta de acesso a serviços de saúde e educação, alimentação inadequada, além das questões relacionadas a condições indevidas de moradia e acesso a serviços de saneamento ${ }^{29}$. Há riscos de futuro agravamento dessas condições de vulnerabilidade, sobretudo em vista das medidas para a contenção da pandemia do COVID-19, tornando as populações mais susceptíveis a manterem o ciclo de doença e pobreza. Políticas públicas que promovam a retirada das pessoas da faixa de pobreza e que alterem de forma positiva os principais determinantes sociais da saúde, como moradia, saneamento, renda e educação, são o melhor antídoto contra os efeitos regressivos da situação social.

Mais especificamente, a inclusão social e as melhorias estruturais que podem advir dessas políticas públicas, desde que gerenciadas de forma adequada, podem permitir a redução dos processos de morbidade por desnutrição e diarreia, e consequentemente da mortalidade por essas causas ${ }^{30}$. Visando perscrutar tendências nesse sentido, a presente revisão sistemática reuniu trabalhos que trazem panoramas e resultados referentes a essas condições de vulnerabilidade e dois achados principais podem ser obtidos das evidências encontradas.

$\mathrm{O}$ primeiro deles refere-se ao PBF. A maior parte dos estudos avaliados nesta revisão mostrou impacto positivo do Programa, relacionado à diminuição dos processos de adoecimento e morte por diarreia e desnutrição em crianças menores de cinco anos de idade. Tais resultados são corroborados por outros estudos que avaliaram o estado nutricional de beneficiários, antes 
e depois da exposição ao Programa e apontaram melhores desfechos em saúde em populações beneficiárias ${ }^{9,12-14,16,18,31,32}$. No entanto, para compreender melhor a ação do PBF torna-se necessária a realização de pesquisas que avaliem, ao mesmo tempo, a cobertura da população total e a cobertura da população alvo pelo Programa, o efeito das suas dimensões e condicionalidades nos resultados de saúde (aumento da renda dos beneficiários, dos anos de escolaridade das crianças e adolescentes beneficiários, futuras mãe (s)/ cuidador(es) de crianças e maior atenção à saúde), além de sua interação com outras políticas públicas.Em particular, a intervenção com saneamento assume grande importância, devido a seu efeito comprovado em melhores resultados de saúde, principalmente relacionados a doenças relacionadas à pobreza que acometem crianças com idade abaixo de cinco anos ${ }^{13,21-23,34,36,38,39}$.

O segundo achado diz respeito ao impacto do saneamento, outro objetivo de análise desta revisão. Independente da forma como a variável de saneamento foi avaliada nos estudos que compuseram a revisão, três dos quatro estudos apontaram efeito protetivo em relação aos processos de adoecimento e morte por diarreia e desnutrição, quando presentes condições adequadas de acesso à água, esgotamento sanitário e/ou coleta de resíduos sólidos. A relação do saneamento com resultados de saúde está bem explicitada na literatura ${ }^{13,21-24,31,33-42}$. No entanto, assim como observado para o PBF, para avaliar o acesso ao saneamento, sabidamente uma política pública atuante nas dimensões social, econômica, política e cultural e com resultados positivos na promoção da qualidade de vida de indivíduos, núcleos familiares e comunidades, torna-se necessária a avaliação de sua interação com outras políticas públicas, como o $\mathrm{PBF}$, assim como sua interação com outras estratégias que objetivam melhorias nas condições de vida e saúde da população brasileira.

De uma forma geral, a tendência mostrada pelos estudos avaliados é evidenciar que melhor acesso, simultaneamente, a PTCR e a condições de saneamento resulta no efeito positivo de cada uma dessas intervenções sobre os desfechos estudados nos estudos, mesmo na presença da outra. Ou seja, cada intervenção contribui com uma parcela de redução das mortes e doenças por diarreia e desnutrição. É possível que a interação entre as duas medidas, não avaliada nos estudos compilados, possa potencializar essa redução. Estudos que avancem nessa compreensão proporcionariam o preenchimento de lacunas na literatura, trazendo evidências que permitam aos gestores dos programas sociais redesenhar programas, com recursos suficientes para alcançar a cobertura de toda a população elegível do PBF e, em conjunto, universalizar o acesso ao saneamento no país.

Para a realização da revisão sistemática, contemplando o efeito do PBF e de intervenções em saneamento nos desfechos morbidade e mortalidade por diarreia e desnutrição em crianças menores de cinco anos de idade, apenas quatro artigos científicos respondiam à pergunta norteadora deste estudo. Como o PBF foi instituído no ano de $2003^{2}$ e intervenções governamentais coordenadas em saneamento são realizadas desde a década de 1950, com marco regulatório instituído no ano de $2007^{6}$, e sendo ambas as políticas relacionadas a desfechos em saúde para grupos semelhantes de doenças e faixas etárias semelhantes, era de se esperar um número maior de estudos publicados contemplando os dois temas, permitindo dessa forma, uma avaliação mais consistente do conjunto de evidências apresentadas.

De toda forma, os resultados sugerem um efeito positivo do PBF e das intervenções em saneamento na diminuição dos processos de adoecimento e morte em crianças menores de cinco anos, pelos desfechos diarreia e desnutrição, doenças essas relacionadas diretamente a pobreza. Embora nenhum estudo tenha tratado da interação entre a exposição da população ao PBF e o acesso ao saneamento adequado, cada uma dessas intervenções, de forma isolada nas interpretações dos resultados dos estudos, se mostrou protetiva. Interessante ressaltar que o efeito do PBF também pôde ser observado por meio do resultado de algumas dimensões do Programa, ou seja, seu efeito protetivo foi observado pelo aumento da renda dos beneficiários, acesso a serviços de saúde e aumento do número de anos de estudo da mãe e/ou chefe da família ${ }^{13,17}$.

Os artigos selecionados para esta revisão apresentaram uma boa qualidade metodológica, segundo critérios adaptados de Downs e Black ${ }^{28}$, garantindo confiabilidade nos seus resultados. Em sua maioria, convergiram para a mesma conclusão, considerando o PBF e a melhoria de serviços de saneamento importantes do ponto de vista da saúde pública.

A presente revisão indica a necessidade de maiores investimentos em estudos que possam avaliar interação do PBF e das intervenções em saneamento. Do ponto de vista das políticas públicas, reforça-se a importância da manutenção 
do PBF e ampliação de sua cobertura, visto que as políticas de austeridade - associadas aos efeitos ainda imprecisos das políticas de contenção da COVID-19 - colocam o risco de um aumento dramático de famílias em situação de pobreza e extrema pobreza no Brasil. Da mesma forma, destaca-se a necessidade demais investimentos em saneamento básico, seguindo as diretrizes do PLANSAB, em prol da universalização do acesso à água e do esgotamento sanitário, metas dos $\mathrm{Ob}$ jetivos do Desenvolvimento Sustentável (ODS 6) a serem alcançadas até o ano de 2030.

\section{Colaboradores}

Todos os autores contribuíram na concepção, planejamento, análise, interpretação e redação do trabalho. Ambos os autores aprovaram a versão final encaminhada.

\section{Financiamento}

Coordenação de Aperfeiçoamento de Pessoal de Nível Superior (CAPES). 


\section{Referências}

1. Shei A. Brazil's Conditional Cash Transfer Program Associated With Declines In Infant Mortality Rates. HealthAffairs 2013; 32(7):1274-1281.

2. Brasil. Lei n. ${ }^{\circ} 10.836$, de 09 de janeiro de 2004. Cria o Programa Bolsa Família e dá outras providências. 2004a.

3. Brasil. Decreto n. ${ }^{\circ} .209$, de 17 de setembro de 2004. Regulamenta a Lei $n^{\circ}$ 10.836, de 9 de janeiro de 2004, que cria o Programa Bolsa Família, e dá outras providências. 2004b.

4. Santos MCS, Delatorre LR, Ceccato MGB, Bonolo PF. Programa Bolsa Família e indicadores educacionais em crianças, adolescentes e escolas no Brasil: revisão sistemática. Cien Saude Colet 2019; 24(6):2233-2247

5. Ministério do Desenvolvimento Social (MDS). Bolsa Família. [Internet] [acessado 2019 maio 24]. Disponível em: https://www.mds.gov.br/bolsafamilia/

6. Brasil. Lei n. ${ }^{\circ} 11.445$, de 05 de janeiro de 2007. Estabelece diretrizes nacionais para o saneamento básico. 2007.

7. Ministério do Desenvolvimento Regional (MDR). Secretaria Nacional de Saneamento (SNS). Plano Nacional de Saneamento Básico - Mais saúde com qualidade de vida e cidadania. Brasília: MDR/SNS; 2019.

8. Agência Nacional de Águas (ANA). OD6 no Brasil: visão da ANA sobre os indicadores/Agência Nacional de Águas. Brasília: ANA; 2019.

9. Silva ESA, Paes NA. Programa Bolsa Família e a redução da mortalidade infantilnos municípios do Semiárido brasileiro. Cien Saude Colet 2019; 24(2):623-630.

10. Silva ESA, Paes NA. Programa Bolsa Família e mortalidade infantil no Brasil: revisão integrativa. HOLOS 2018; 34(1):201-211.

11. Rasella D, Aquino R, Santos CAT, Paes-Sousa R, Barreto ML. Effect of a conditional cash transfer programme on childhood mortality: a nationwide analysis of Brazilian municipalities. The Lancet 2013;6:57-64.

12. Pinho Neto VR, Berriel CM. Transferências Condicionais de Renda e Nutrição: Efeitos do Bolsa Família nas áreas rurais e urbanas do Brasil. Economia Aplicada 2017; 21(2):185-205

13. Imada KS, Araújo TS, Muniz PT, Pádua VL. Fatores socioeconômicos, higiênicos e de saneamento na redução de diarreia na Amazônia. Rev Saude Publica 2016; 50(77):1-11.

14. Saldanha LF, Lagares EB, Fonseca PC, Anastácio LR. Estado nutricional de crianças beneficiárias do Programa Bolsa Família acompanhadas pelo Sistema de Vigilância Alimentar e Nutricional no Estado de Minas Gerais. Ver Med Minas Gerais 2014; 24(4):478-485.

15. Chagas DC, Silva AAM, Batista RFL, Simões VMF, Lamy ZC, Coimbra LC, Alves MTSSB. Prevalência e fatores associados à desnutrição e ao excesso de peso em menores de cinco anos nos seis maiores municípios do Maranhão. Rev Bras Epidemiol 2013;16(1):146-56.

16. Paula DV, Botelho LP, Zanirati PF, Lopes ACS, Santos LC. Avaliação nutricional e padrão de consumo alimentar entre crianças beneficiárias e não beneficiárias de programas de transferência de renda, em escola municipal do Município de Belo Horizonte, Estado de Minas Gerais, Brasil, em 2009. Epidemiol Serv Saude 2012; 21(3):385-394.

17. Paes-Sousa R, Santos LMP, Miazaki LS. Effects of a conditional cash transfer programme on child nutrition in Brazil. Bulletin of the World Health Organization 2011; 89(7):496-503.
18. Camelo RS, Tavares PA, Saiani CCS. Alimentação, Nutrição e Saúde em Programas de Transferência de Renda: Evidências para o Programa Bolsa Família. Economia 2009; 10(4):685-713.

19. Faria LAN, Pena APSA. Análise do impacto do programa bolsa família em relação ao estado nutricional de crianças de zero a sete anos pertencentes às famílias beneficiárias do programa no município de Pará de Minas, Minas Gerais, no período de 2007/2008. SynThesis Revista Digital FAPAM 2009; 1(1):344-354.

20. Fonseca JE, Carneiro M, Pena JL, Colosimo EA, Silva NB, Costa AGFC, Moreira LE, Cairncross S, Heller L. Reducing Occurrence of Giardia duodenalis in Children Living in Semiarid Regions: Impact of a Large Scale Rainwater Harvesting Initiative. PLoS Neglected Tropical Diseases 2014; 8(6):e2943.

21. Teixeira JC, Gomes MHR, Souza JA. Análise da associação entre saneamento e saúde nos estados brasileiros - estudo comparativo entre 2001 e 2006. EngSanit Ambient 2011; 16(2):197-204. -204. [acessado 2020 jan 10]. Disponível em: https://doi.org/10.1590/ S1413-41522011000200014.

22. Silva RR, Silva CA, Jesus Pereira CA, Carvalho NRL, Negrão-Correa D, LamounierJÁ, Carneiro M. Association between nutritional status, environmental and socio-economic factors and Giardia lamblia infections among children aged 6-71 months in Brazil. Trans R Soc Trop Med Hyg 2009; 103(5):512-9.

23. Teixeira JC, Heller L, Barreto ML. Giardia duodenalis infection: risk factors for children living in sub-standard settlements in Brazil (Research Note). Cad Saude Publica 2007; 23(6):1489-1493.

24. Barreto ML, Genser B, Strina A, Teixeira MG, Assis AMO, Rego RF, Teles CA, Prado MS, Matos SM, Santos DN, Santos LA, Cairncross S. Effect of city-wide sanitation programme on reduction in rate of childhood diarrhoea in northeast Brazil: assessment by two cohort studies. The Lancet 2007; 370(9599):1622-1628.

25. Higgins JPT, Green S. Cochrane handbook for systematic reviews of interventions 5.1.0 [updated March 2011]. London: The Cochrane Collaboration; 2011.

26. Liberati A, Altman DG, Tetzlaff J, Mulrow C, Gotzsche PC, Ioannidis JP, Clarke M, Devereaux PJ, Kleijnen J, Moher D.The PRISMA statement for reporting systematic reviews and meta-analyses of studies that evaluate healthcare interventions: explanation and elaboration. BMJ 2009; 6(7):1-28.

27. Whttemore R, Knaff K. The integrative review: update methodology. J Adv Nurs 2005; 52(5):546-553.

28. Downs SH, Black N. The feasibility of creating a checklist for the assessment of the methodological quality both of randomised and non-randomised studies of health care interventions. J Epidemiol Community Health 1998; 52(6):377-384.

29. Alves H, Escorel S. Processos de exclusão social e iniquidades em saúde: um estudo de caso a partir do Programa Bolsa Família, Brasil. Rev Panam Salud Publica 2013; 34(6):429-436.

30. Araújo-Jorge T, Matraca M, Neto AM, Trajano V, Andrea P, Fonseca A. Doenças negligenciadas, erradicação da pobreza e o plano Brasil sem miséria. $1^{\text {a }}$ ed. Brasília: Ministério do Desenvolvimento Social e Combate à Fome; 2014. 
31. Buhler HF, Ignotti E, Neves SMAS, Hacon SS. Spatial analysis of integrated health and environmental indicators for morbidity and mortality due to infant diarrhea in Brazil, 2010. Cad Saude Publica 2014; 30(9):1921-1934.

32. Guanais F. Efectos combinados de laampliación de laatención primaria de salud y de lastransferencias condicionadas de dinero em efectivo sobre lamortalidad infantil en Brasil,1998-2010. Rev Panam Salud Publica 2014; 36(1):65-72.

33. Bartram J, Cairncross S. Hygiene, Sanitation, and Water: Forgotten Foundations of Health. PLoS Medicine 2010; 7(1):1-9. [acessado 2020 jan 10]. Disponível em: https://doi.org/10.1371/journal.pmed.1000367.

34. Mokdad AH. Estimates of global, regional, and national morbidity, mortality, and aetiologies of diarrhoeal diseases: a systematic analysis for the Global Burden of Disease Study 2015. Lancet InfectDis 2015; 17(9):909-948.

35. Cowman G, Otipo S, Njeru I, Achia T, Thirumurthy H, Bartram J, Kioko J. Factors associated with cholera in Kenya, 2008-2013. Pan African Medical Journal 2017; 28:101.

36. Assefa S, Hailu D, Kabeta A, Berhanu G. Household sanitation practice associated with nutritional status of pre-school children aged 24-59 months in Hawassa Zuria Woreda, South Ethiopia: A cross-sectional study. Current Pediatri cResearch 2017; 21(2):291-297.

37. Khalil I, Colombara DB, Forouzanfar MH, Troeger C, Daoud F, Moradi-Lakeh M, Khalil I, Colombara DV, Forouzanfar MH, Troeger C, Daoud F, Moradi-Lakeh M, Bcheraoui CE, Rao PC, Afshin A, Charara R, Abate KH, Razek MMAE, Abd-Allah F, Abu-Elyazeed R, Kiadaliri AA, Akanda AS, Akseer N, Alam K, Alasfoor D, Ali R, AlMazroa MA, Alomari MA, Al-Raddadi RMS, Alsharif U, Alsowaidi S, Altirkawi KA, Alvis-Guzman N, Ammar W, Antonio CAT, Asayesh H, Asghar RJ, Atique S, Awasthi A, Bacha U, Badawi A, Barac A, Bedi $\mathrm{N}$, Bekele T, Bensenor IM, Betsu BD, Bhutta Z, Abdulhak AAB, Butt ZA, Danawi H, Dubey M, Endries AY, Faghmous IDA, Farid T, Farvid MS, Farzadfar F, Fereshtehnejad SM, Fischer F, Fitchett JRA, Gibney $\mathrm{KB}$, Ginawi IAM, Gishu MD, Gugnani HC, Gupta R, Hailu GB, Hamadeh RR, Hamidi S, Harb HL, Hedayati MT, Hsairi M, Husseini A, Jahanmehr N, Javanbakht M, Jibat T, Jonas JB, Kasaeian A, Khader YS, Khan AR, Khan EA, Khan G, Khoja TAM, Kinfu Y, Kissoon N, Koyanagi A, Lal A, Latif AAA, Lunevicius R, Razek HMAE, Majeed A, Malekzadeh R, Mehari A, Mekonnen AB, Melaku YA, Memish ZA, Mendoza W, Misganaw A, Mohamed LAI, Nachega JB, Nguyen QL, Nisar MI, Peprah EK, Platts-Mills JA, Pourmalek F, Qorbani M, Rafay A, Rahimi-Movaghar V, Rahman SU, Rai RK, Rana SM, Ranabhat CL, Rao SR, Refaat AH, Riddle M, Roshandel G, Ruhago GM, Saleh MM, Sanabria JR, Sawhney M, Sepanlou SG, Setegn T, Sliwa K, Sreeramareddy CT, Sykes BL, Tavakkoli M, Tedla BA, Terkawi AS, Ukwaja K, Uthman OA, Westerman R, Wubshet M, Yenesew MA, Yonemoto N, Younis MZ, Zaidi Z, Zaki MES, Rabeeah AAA, Wang H, Naghavi M, Vos T, Lopez AD, Murray CJL, Mokdad AH.et al. Burden of Diarrhea in the Eastern Mediterranean Region, 1990-2013: Findings from the Global Burden of Disease Study 2013. Am J Trop Med Hyg 2016; 95(6):1319-1329.
38. Mukabutera A, Thomsom D, Murray M, Basinga P, Nyirazinyoye L, Atwood S, Savage KP, Ngirimana A, Hedt-Gauthier BL. Rainfall variation and child health: effect of rainfall on diarrhea among under 5 children in Rwanda, 2010. BMC publichealth 2016; 16(731):19. [acessado 2020 jan 10]. Disponível em: https://doi. org/10.1186/s12889-016-3435-9.

39. Deribew A, Tessema GA, Deribe K, Melaku YA, Lakew Y, Amare AT, Abera SF, Mohammed M, Hiruye A, Teklay E, Misganaw A, Kassebaum N. Trends, causes, and risk factors of mortality among children under 5 in Ethiopia, 1990-2013: findings from the Global Burden of Disease Study 2013. Population Health Metrics 2016; 14(42):1-10.

40. Njuguna C, Njeru I, Mgamb E, Langat D, Makokha A, Ongore D,Mathenge E, Kariuki S. Enteric pathogens and factors associated with acute bloody diarrhoea, Kenya. BMC Infectious Diseases 2016; 16(477):1-10.

41. Fuller JA, Westphal JA, Kenney B, Eisenberg JN. The joint effects of water and sanitation on diarrhoeal disease: a multicountry analysis of the Demographic and Health Surveys. Trop Med Int Health 2015; 20(3):284-292.

42. Ezek OK. Agho KE, Dibley MJ, Hall J, Page AN. The impact of water and sanitation on childhood mortality in Nigeria: evidence from demographic and health surveys, 2003-2013. Int J Environ Res Public Health 2014; 11(9):9256-9272.

Artigo apresentado em 16/01/2020

Aprovado em 17/06/2020

Versão final apresentada em 19/06/2020

Editores-chefes: Romeu Gomes, Antônio Augusto Moura da Silva 